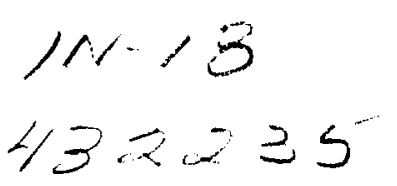

\title{
EXPRESS Service to the International Space Station: EXPRESS Pallet
}

\author{
Lowell Primm ${ }^{1}$ and Alan Bergmann ${ }^{2}$ \\ ${ }^{1}$ Space Station Utilization Office, Mail Code JA62, NASA-Marshall Space Flight Center, Huntsville, AL 35812 \\ ${ }^{2}$ Boeing Defense and Space Group, EXPRESS Pallet Project Office, PO Box 240002 Huntsville Al 35824-6402
}

\begin{abstract}
The International Space Station (ISS) will be the ultimate scientific accomplishment in the history of NASA, with its primary objective of providing unique scientific investigation opportunities. This objective is the basis for the creation of the EXPRESS Pallet System (ExPS). The EXPRESS Pallet will provide external/unpressurized accommodations for a wide variety of external users. The payload developers represent many science disciplines, including earth observation, communications, solar and deep space viewing, long-term exposure, and many others. The EXPRESS Pallet will provide a mechanism to maximum utilization of the limited ISS unpressurized payload volume, standard physical payload interfaces for users, a standard integration template for users and the capability to changeout payloads on-orbit. The EXPRESS Pallet provides access to Ram, Wake, Starboard, Port, Nadir, Zenith and Earth Limb for exposure and viewing. The ExPs consists of the Pallet structure, payload Adapters, and a subsystem assembly which includes data controller, power distribution and conversion, and Extra Vehicular Robotics/ExtraVehicular Activity systems.
\end{abstract}

\section{HISTORY}

In 1988, NASA established accommodations for 13 external US payloads on Space Station. In 1990, the Space Station Program eliminated the attached payload capability. In the following two years, users continually identified the critical need for attached payload accommodations, which resulted in an accommodation study that was conducted by the Space Station Program in 1993. Included among the results of the study was the need for a simple, modular external payload carrier system. In 1994, the EXPRESS Pallet feasibility study was performed, and the Attached Payload Working Group (APWG) and EXPRESS Pallet Analysis and Integration Team were formed. Finally, in 1995, full funding by International Space Station was initiated for the EXPRESS Pallet Program.

\section{THE EXPRESS PALLET PHILOSOPHY}

The EXPRESS Pallet is a part of the EXpedite the PRocessing of Experiments to Space Station (EXPRESS) Program. This program, which applies to internal (Rack) and external experiment hardware, is putting users on the fast track to Space Station, by providing a shortened integration cycle for standard payloads and providing standard, modular system accommodations and services. EXPRESS Pallet is the primary ISS carrier for external payloads, and its purpose is to provide rapid, simplified integration of attached payloads to ISS. The goal of the EXPRESS program is to ultimately provide a capability to integrate and fly payloads within Launch-11 months.

\section{MISSION OVERVIEW}

The ExPS will be transported to and from orbit via the Space Shuttle, using standard keel and longeron attachments. The ExPS is robotically removed from the Shuttle cargo bay and attached to the S3 or P3 truss segment via the Payload Attach System (PAS). ExPS payloads will have access to Ram, Wake, Starboard, Port, Nadir, Zenith and Earth Limb for exposure and viewing. Payloads are operated using standard ExPS interfaces available at each EXPRESS Pallet Adapter (ExPA) location. The standard interfaces include power and data, delivered to each Adapter/payload identically. Once the ExPS is on orbit, replacement ExPAs will periodically be transported to orbit via an ISS Unpressurized Logistics Carrier/sidewall carrier; routine changeout of the ExPAs will be accomplished 
using the ISS dexterous robotic arm, referred to as the Special Purpose Dexterous Manipulator. The capability to routinely changeout payloads/Adapters is key to the long-term viability of the ExPS.

\section{PAYLOAD INTEGRATION}

The philosophy of the EXPRESS concept is streamlined integration. By establishing standard flight system accommodations, the payload integration process is reduced in complexity, which allows payloads to be integrated as late as Launch-11 months during steady-state flight operations.. The payload developer will be provided standard documentation, including a Payload Accommodations Handbook (PAH) an Interface Definition Document (IDD), and a generic Payload Verification Plan (PVP) which are the centerpieces for the integration process. The PAH provides the user a system description, accommodations, and detailed information on payload integration processes, as examples. The IDD is the payload-to-EXPRESS Pallet interface requirements document and consists of elements including structural loads characteristics, thermal environment, field-of-view, electrical interfaces, etc. The PVP provides the user with a "blank book" listing the required verification data that must be supplied by payload developers for flight.

The payload developer has increased participation in the ISS payload integration and verification process. The user will be supplied an EXPRESS Pallet Adapter, which serves as the structural and electrical interface to each payload. In addition, users will be provided portable computers, called Suitcase Test Environment for Payloads-EXPRESS Pallet (STEP-EP), which is a tool for verifying payload C\&DH interfaces to EXPRESS Pallet. The STEP-EPs are portable computers containing software which emulates the functions of the EXPRESS Pallet flight system. STEPEPs will be loaned to users up to 18 months prior to flight. The user is responsible for physically integrating their payloads onto the Adapter, verifying their interfaces using the STEP-EP, developing required safety data, and shipping the integrated Adapter mounted payload to KSC. This "ship-and-shoot" concept is key to the success of the EXPRESS philosophy. At KSC, the Adapters/payloads are physically integrated onto the Pallet system, or the Unpressurized Logistics Carrier. Payloads will be integrated onto the ULC for payload changeouts after the initial Pallets are launched.

As previously discussed, Brazil's INPE will provide flight and ground system hardware for EXPRESS Pallet; however, payload analytical integration activities are centered at NASA's Marshall Space Flight Center (MSFC). The EXPRESS Pallet project at MSFC provides Adapter-to-Adapter analytical integration services, payload complement integration and verification, and safety data packages for the entire Pallet payload complement. In addition, the project provides the critical interface between payload developers and ISS, and provides users with accommodations assessments, as required. For most payload developers, the project's EXPRESS Pallet Engineering Integration Managers will be the key interface for flight on-board the ISS.

\section{PAYLOAD OPERATIONS}

Payloads may be operated while on-orbit by several means: using the Space Station's Payload Operations Integration Center, with the crew using a portable laptop, and utilizing payload developers remote sites. To date, most payload developers are using their remote sites as the primary operation site. The ExPS controller is capable of providing the critical link between the ISS systems and a user's payload. In addition, a capability has been developed at MSFC which is referred to as the Payload Operations Integration Function (POIF). The POIF includes payload operations ground controllers and crew and ground training personnel, and develops payload operations requirements.

\section{ISS ATTITUDE}

The ISS flies in a fixed attitude with respect to Earth. The ISS truss is parallel with the surface of the Earth and perpendicular to the velocity vector, which provides a stable, oriented platform with only a few degrees of deviation due to variations in aerodynamic torques from atmospheric drag. The orbital inclination is 51.6 degrees and the 
altitude varies from about 330 to $460 \mathrm{~km}$ (180 to 250 nautical miles) due to orbital decay and reboost thruster firings.

\section{PRIME ITEM DIAGRAMS}

The ExPS interface diagram is presented in Figure 1. The physical diagram for the ExPS is presented in Figure 2.

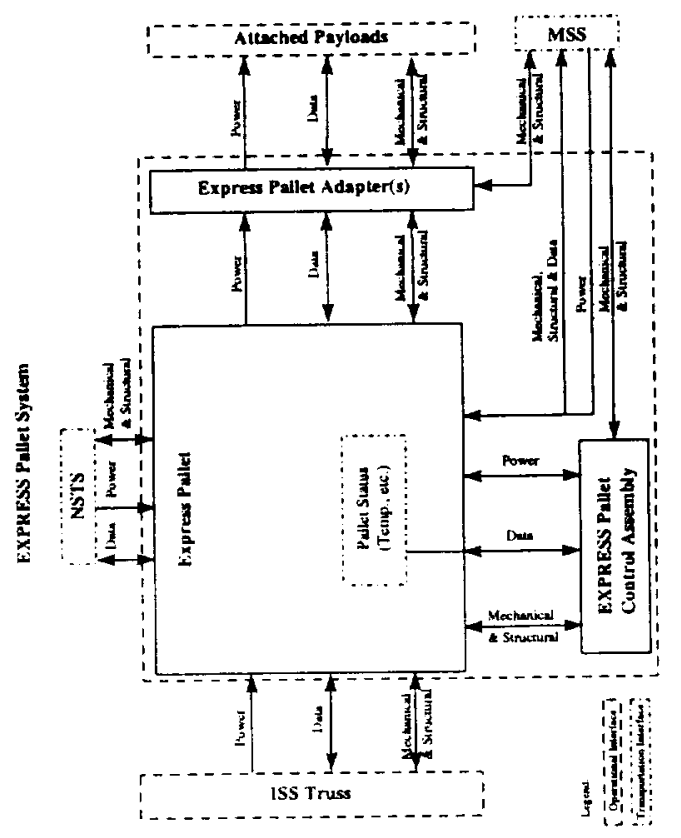

FIGURE 1. EXPRESS Pallet Interface Diagram

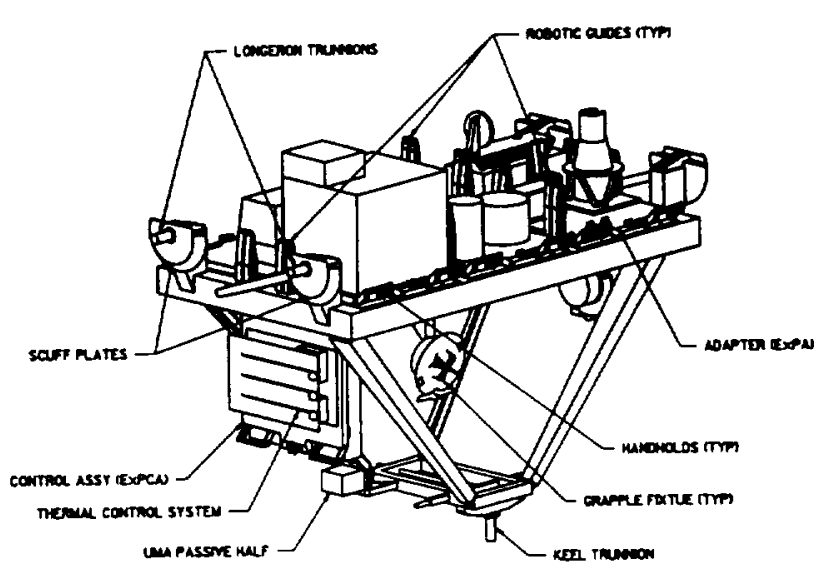

FIGURE 2. EXPRESS Pallet Isometric View

\section{SYSTEM DESCRIPTION}

The EXPRESS Pallet System (ExPS) is the International Space Station (ISS) truss attached payload facility which provides standard ISS accommodations for multiple external Express Pallet Adapter (ExPA) mounted payloads. It accommodates on-orbit installation, removal, and change-out of ExPA mounted payloads, using ISS standard logistics and extravehicular robotics (EVR) capability. Although EVR is the program baseline for EXPRESS Pallet, contingency extravehicular activities (EVA) can be utilized.

The ExPS is composed of three main functional elements: ExPAs, the Express Pallet (ExP), and EXPRESS Pallet Control Assembly (ExPCA). The ExPA provides standard structural, mechanical, electrical and communications interfaces for payloads. The ExPA is capable of providing for a structural and mechanical interface with the ExP, with a capability for robotics or EVA manipulation, connection and attachment of Adapter-to-Pallet related interfaces. The ISS program baseline requires EVR operations only; contingency EVA occurs only upon failure or unavailability of EVR. The ExPA will utilize the ISS logistics systems and is capable of providing stay alive power to payloads - distributed by the ExPCA from a connection made between the ExP and the Orbiter while in the Orbiter cargo bay and while the ExPS is attached to the MSS enroute to its final destination on the ISS Truss.

The ExP provides a structural platform for the integration of ExPA mounted payloads. It provides for a structural and mechanical interface with the ISS Truss Site (S3/P3), with a capability for EVR or EVA manipulation, connection, and attachment of structural, electrical, and data interfaces. 
The ExPCA provides data/command routing, status monitoring, and power control, distribution, and conversion. Up to 6 ExPAs will be directly serviced by this assembly. The ExPCA is EVR and EVA compatible for manipulation, connection and replacement.

\section{GENERAL SYSTEM REQUIREMENTS}

The EXPRESS Pallet is built based on the concept of a continuous operating life cycle, i.e., fully integrated pallets will be transported to orbit and changeout of individual payloads will be necessary during the pallet's life. The ExPS will be able to accommodate user site integration/de-integration of payloads to Adapters $\mathrm{n}$ addition to on-ground integration/de-integration of payload adapters to the pallet or ULC at the KSC launch site. An ExPS simulator to support pre-flight check out of integrated Pallet payload operations will be developed and located at KSC. The Payload Attachment System (PAS), located on the S3/P3 Truss Sites, will provide the active half of the Umbilical Mechanism Assembly (UMA) interface to the ExPS to allow access the ISS Command and Data Handling (C\&DH) and power systems.

\section{Operational Lifetime}

The EXPRESS Pallet design is predicated on the following considerations. The ExPS will be required to operate for 10 years given periodic inspections, preventive and corrective maintenance, restoration, replacement of components, and re-supply operations. As previously discussed, the preferred scenario for flight operations of the EXPRESS Pallet is to transport a fully integrated Pallet to orbit and provide routine changeout of individual payloads based on user requirements. As a result, ExPS major components are being designed to withstand the following minimum set of launch and return missions: ExP -- 10 launch/landing missions, ExPA -- 10 launch/landing missions, and ExPCA - 5 launch/landing missions. The EXPRESS Pallet avionics are designed with a Mean Time Between Failure of 25000 hours.

\section{EXPRESS Pallet Accommodations}

The set of standard services for payload accommodations are listed below.

TABLE 1. EXPRESS Pallet Accommodations.

\begin{tabular}{|c|c|}
\hline Type Accommodation & Total Pallet Resource Allocation \\
\hline Structure & 6 adapters per pallet \\
\hline Payload Weight & $500 \mathrm{lbs}$ maximum per adapter with CG described below \\
\hline Payload Envelope & 34 inches width $\times 46$ inches length $\times 49$ inches height \\
\hline Power* & $\begin{array}{l}2.5 \mathrm{~kW} \text { capability at } 120 \mathrm{Vdc}(2 \text { feeds) } 1.25 \mathrm{~kW} \text { per feed } \\
1000 \mathrm{~W} \text { capability at } 28 \mathrm{Vdc} \text { ( } 2 \text { feeds) } 500 \mathrm{~W} \text { per feed }\end{array}$ \\
\hline $\begin{array}{l}\text { Command, Control, Data, and } \\
\text { Telemetry }\end{array}$ & $\begin{array}{l}\text { MIL-STD- } 1553 \text { Bus ( } 2 \text { interfaces at each ExPA) } \\
\text { Analog ( } 6 \text { Input only ) } \\
\text { Discrete ( } 6 \text { programmable } \text { VO) } \\
\text { Two Ethernet Interfaces }(10 \text { base T) } \\
\end{array}$ \\
\hline Stay-alive & $\begin{array}{ll}\text { Orbiter Bay }-120 \mathrm{vdc} \\
& 28 \mathrm{vdc} \quad 120 \text { watts } \\
\text { MBS - } & 120 \mathrm{vdc} \\
& 28 \mathrm{vdc} \text { TBD watts } \\
\end{array}$ \\
\hline Thermal Control & Passive (Each payload will provide own thermal control) \\
\hline Field of View & Nadir (Earth) or Zenith (Ram, Wake, or Earth Limb are also possible) \\
\hline
\end{tabular}

*Note: The total maximum power to the EXPRESS Pallet is $3 \mathrm{KW}$ at $120 \mathrm{vdc}$ interface $\mathrm{c}$. However, due to resource limitations for the ISS, the total power available to a single pallet will be less. The power is allocated to the EXPRESS Pallet during the mission planning process. 


\section{Payload Mass and Center of Gravity(CG)}

As stated in Table 1, the EXPRESS Pallet payload will be designed to accommodate a maximum payload weight of $500 \mathrm{lb} .(227 \mathrm{~kg}$ ) at each adapter location with a center of gravity (c.g.) as shown in the table below.

TABLE 2. Payload Mass and Center of Gravity(CG).

\begin{tabular}{|l|l|l|}
\hline Payload Mass (lb.) & $\begin{array}{l}\text { Maximum Deviation from Geometric } \\
\text { Center in } \mathrm{X}_{0}-\mathrm{Y}_{0} \text { plane (in) }\end{array}$ & $\begin{array}{l}\text { Maximum Height (Z0) of Payload } \\
\text { Center of Gravity (CG) Above the } \\
\text { ExPA Mounting Plane (in.) }\end{array}$ \\
\hline $401-500$ & $\mathrm{EX}_{0}=7.5, \mathrm{EY}_{0}=7.5$ & 19.5 \\
\hline $301-400$ & $\mathrm{EX}_{0}=9, \Subset \mathrm{EY}_{0}=10$ & 24.0 \\
\hline $201-300$ & $\mathrm{EX}_{0}=10.5, \Subset \mathrm{EY}_{0}=12$ & 28.0 \\
\hline$<200$ & $\Subset \mathrm{EX}_{0} 12, \Subset \mathrm{EY}=14$ & 30.0 \\
\hline
\end{tabular}

\section{Payload Dimensional Envelope}

The EXPRESS Pallet is designed to accommodate six payload adapters with the following form factor maximum dimensions for the payload installed on an adapter:

$$
\begin{array}{ll}
\text { Length } & 46 \mathrm{in} .(116.84 \mathrm{~cm}) \\
\text { Width } & 34 \mathrm{in} .(86.36 \mathrm{~cm}) \\
\text { Height } & 49 \mathrm{in} .(124.46 \mathrm{~cm})
\end{array}
$$

This will provide users with approximately $1564 \mathrm{sq}$. in. of mounting surface and $76636 \mathrm{cu}$. in. of volume for exclusive experiment use.

\section{Command, Control, Data and Telemetry Requirements}

The EXPRESS Pallet avionics provides a variety of data interface options to the user. For commanding the payload; regardless of whether the command source is the crew, Payload Operations Integration Center, the user's home site or the automated procedure executor on board the station; the EXPRESS Pallet is able to receive commands and either process them or act as a data router for the commands. Additionally, the avionics are capable of receiving service requests from the payload for utilization of preplanned ISS services. The EXPRESS Pallet avionics is a remote terminal on the payload MDM and serves as the bus controller for the 1553 local bus on the pallet. In addition to commands and service requests, the EXPRESS Pallet 1553 will periodically collect health/status, safety, and low rate telemetry data from the payloads. This data will be transmitted to the payload MDM for processing or transmitted to the ground. An additional service offered by the ISS data system is the distribution of ancillary data via a MIL-STD-1553 interface.

The EXPRESS Pallet avionics also offer a discrete input monitoring capability at ExPA locations with electrical characteristics of $0-5 \mathrm{vdc}+/-15 \%$ or $0-20 \mathrm{vdc}+/-15 \%$ and discrete output control capability at ExPA locations of 0 $5 \mathrm{vdc}+/-15 \%$ or $0-28 \mathrm{vdc}+/-15 \%$.. Analog monitoring capability is provided at ExPA locations with electrical characteristics compatible with 100 to $1 \mathrm{~K}$ ohm RTDs, 4-20 mA pressure sensors and $+/-5$ vdc differential voltage interfaces.

The EXPRESS Pallet avionics are capable of supporting aggregate data rates up to $250 \mathrm{kbps}$ on the MIL-STD-1553 interface from ExPA locations. A data rate up to $250 \mathrm{kbps}$ will be supported for Payload Local Bus transfers to and from the ISS. 
The EXPRESS Pallet avionics provides IEEE 802.3 Ethernet 10 Base-T interfaces to each ExPA location, accommodating up to $6 \mathrm{Mbps}$ from one ExPA source at a time and transfer this data to the ISS high rate data interface for support of payload science data.

\section{Stay Alive Power Requirements}

As stated in Table 2, the EXPRESS Pallet is capable of distributing stay-alive power during the transportation phase. Orbiter-provided $120 \mathrm{vdc}$ stay-alive power and MIL-STD-1553B data/commands will be routed to the ExPCA and ExPA locations while the orbiter bay doors are open. Additionally, a limited capability to provide 28 vdc stay-alive power, derived from Orbiter-provided $120 \mathrm{vdc}$ power, will be provided.

Once removed from the orbiter bay and mounted on the Mobile Base System (MBS), the ExPCA is capable of distributing $120 \mathrm{vdc}$ stay-alive power and MIL-STD-1553B data/commands (at MSB worksite locations) to Adapter mounted payloads. Additionally, a limited capability to convert to $28 \mathrm{vdc}$ stay-alive power while attached to the MBS per SSP 42004, Section B. will be provided.

\section{CONCLUSION}

The EXPRESS Pallet provides unique opportunities for ISS external payloads. Using a standardized, modular design, the Pallet maximizes use of the limited ISS external payload locations. The Pallet's capability for providing power, data, and payload changeout capability is critical to the success of the external payload community.

The streamlined payload integration process provides for a rapid payload turnaround (from launch to orbit to return), with increased participation by payload developers. This increased participation is offset by more standardized processes and documentation. In addition, users now have the opportunity for remote payload operations at their home facility.

The EXPRESS Pallet system meets the key goal of NASA projects: faster, better, cheaper. Payloads are integrated faster using the EXPRESS philosophy; standardization provides a better method for accommodating payload requirements; costs for payloads flying to ISS will be cheaper due to streamlined integration and operations activities. 